\title{
Workflow for the Targeted and Untargeted Detection of Small Metabolites in Fish Skin Mucus
}

\author{
Lada Ivanova ${ }^{1, *}$, Haitham Tartor ${ }^{2}$ (D), Søren Grove ${ }^{2}$, Anja B. Kristoffersen ${ }^{3}$ and Silvio Uhlig ${ }^{1}$ \\ 1 Chemistry Section, Norwegian Veterinary Institute, P.O. Box 750, Sentrum, N-0106 Oslo, Norway; \\ silvio.uhlig@vetinst.no \\ 2 Fish Health Research Group, Norwegian Veterinary Institute, P.O. Box 750, Sentrum, N-0106 Oslo, Norway; \\ haitham.tartor@vetinst.no (H.T.); soren.grove@vetinst.no (S.G.) \\ 3 Epidemiology Section, Norwegian Veterinary Institute, P.O. Box 750, Sentrum, N-0106 Oslo, Norway; \\ anja.kristoffersen@vetinst.no \\ * Correspondence: lada.ivanova@vetinst.no; Tel.: +47-23-21-6119
}

Received: 9 May 2018; Accepted: 31 May 2018; Published: 7 June 2018

\begin{abstract}
The skin mucus of fish is in permanent contact with the aquatic environment. Data from the analysis of the chemical composition of skin mucus could potentially be used for monitoring the health status of the fish. Knowledge about mucus composition or change in composition over time could also contribute to understanding the aetiology of certain diseases. The objective of the present study was the development of a workflow for non-invasive sampling of skin mucus from farmed salmon (Salmo salar) for the targeted and untargeted detection of small metabolites. Skin mucus was either scraped off, wiped off using medical wipes, or the mucus' water phase was absorbed using the same type of medical wipes that was used for the wiping method. Following a simple filtration step, the obtained mucus samples were subjected to hydrophilic interaction chromatography coupled to high-resolution mass spectrometry. Post-acquisition processing included the targeted analysis of 86 small metabolites, of which up to 60 were detected in absorbed mucus. Untargeted analysis of the mucus samples from equally treated salmon revealed that the total variation of the metabolome was lowest in absorbed mucus and highest in the scraped mucus. Thus, future studies including small-molecule metabolomics of skin mucus in fish would benefit from a sampling regime employing absorption of the water phase in order to minimize the bias related to the sampling step. Furthermore, the absorption method is also a less invasive approach allowing for repetitive sampling within short time intervals.
\end{abstract}

Keywords: skin mucus; salmon; sampling regime; metabolomics; Salmo salar

\section{Introduction}

An increasing world population size, standard of living, and globalization have placed excessive demands on the aquaculture industry that according to the Food and Agriculture Organization of the United Nations needs to double food production by mid-century [1]. The economic success of the modern aquaculture is intimately attributed to the health and welfare of the aquatic animals [2]. In this context, mucus plays a central role in maintaining fish health providing a physical and biochemical barrier against a broad spectrum of pathogens present in aquatic environments [3]. The implication of fish skin mucus in the immune response and disease resistance has been well documented during the last decades, in addition to its involvement in respiration, ionic and osmotic regulation, excretion, reproduction and locomotion [2-5]. Fish skin mucus was demonstrated to be a source for numerous immune-related components such as lysozymes, phosphatases, esterases, proteolytic enzymes, complement factors, lectins, immunoglobulins, C-reactive proteins, 
and lymphocytes [2,6,7]. Additionally, an antibacterial effect of fish skin mucus was reported in several species [8,9]. The observed antibacterial activity was stronger toward Gram positive compared to Gram negative bacteria, but the key bioactive components have not yet been identified. Thus, these findings highlight that fish skin mucus is a rich and yet relatively unexplored source of bioactive molecules. Studying the mucus metabolome is therefore important for the identification of the bioactive compounds as well as effective biomarkers for fish health status. Recently, 204 low molecular weight molecules were detected and tentatively identified in the skin mucus collected from the fathead minnow (Pimephales promelas) by using liquid chromatography-tandem mass spectrometry (LC-MS/MS) [10]. Such "metabolic signature" might be utilized as a starting point for a better understanding of mucus functionality and dynamics in disease progression.

Mucus has recently received significant interest since it may be collected in a simple and non-invasive manner thereby enabling the analysis and monitoring of molecules of interest over time providing relevant information about the health status of the fish [10]. However, the sample collection technique may be an important source for bias in comparative metabolomics of fish skin mucus [10]. Our objective was therefore to use high-resolution mass spectrometry-based targeted and untargeted metabolomics for the comparison of three different sampling procedures in order to find the least biased and most robust sampling strategy and subsequent workflow.

\section{Results and Discussion}

The selection of the 86 metabolite standards for the targeted analysis of fish skin mucus was based on a recent metabolite profiling study [10]. In that study, metabolites were putatively identified based on their mass spectrometric characteristics with reference to the Human Metabolome Database. The annotated metabolites were all low-molecular weight, polar compounds, which in our experience are best separated prior to mass spectrometric detection using hydrophilic interaction chromatography (HILIC). A polymeric zwitterionic separation column, eluted with a basic $\mathrm{pH}$ mobile phase, was recently shown to perform well for the separation of small metabolites [11]. In the present study, the same column and mobile phase was also well suited for chromatography of the 86 target metabolites, and working standards for each metabolite were initially run in order to establish retention times and mass spectrometric characteristics (Table S2) as well as high-resolution tandem mass spectrometry (HRMS/MS ) characteristics.

\subsection{Sample Preparation for Targeted Liquid Chromatography-High-Resolution Mass Spectrometry Analysis of Fish Skin Mucus Metabolites}

Skin mucus was scraped from 10 fish and pooled in order to investigate the presence of the 86 target metabolites using five sample treatment protocols. Preparation method (PM) 5 was a straightforward filter-and-shoot approach, which allowed for detection of the highest number of metabolites (56, Table 1).

It was also the sample pretreatment method with the highest reproducibility, yielding the lowest relative standard deviations (average 8\%) for individual peak areas of the detected metabolites (Table 1). We thus chose PM 5 for the subsequent analyses of skin mucus samples. In a few cases, the extracted ion chromatograms showed a major peak at a retention time that was substantially different from that of the reference compound, but with identical $\mathrm{m} / \mathrm{z}$ and even similar HRMS/MS product ion spectrum. These were 3 -furoic acid, $N$-acetyl-L-alanine, pyrrolidone, 4-aminobenzoic acid, erythronic acid, adenosine-3'-monophosphate, $N$-acetylneuraminic acid, L-histidine, $O$-phosphoethanolamine, cytidine- $5^{\prime}$-monophosphate, guanosine- $5^{\prime}$-monophosphate, $\mathrm{N}$-acetylputrescine hydrochloride, piperidine, and urea. Some of these metabolites could be expected to be constituents of mucus, e.g., $N$-acetylneuraminic acid. However, as the observed liquid chromatography-high-resolution mass spectrometry (LC-HRMS) peaks did not coincide with the reference compounds, they were not included in the targeted metabolite data set. These results also 
show that putative database annotation could lead to misidentification and warrants the need for further confirmation by reference standards [10].

Table 1. Number of metabolites detected using targeted Liquid Chromatography-High-Resolution Mass Spectrometry (LC-HRMS) and repeatability of sample preparation methods evaluated as relative standard deviations of peak areas from triplicate analyses.

\begin{tabular}{cccc}
\hline PM & $\begin{array}{c}\text { Number of } \\
\text { Detected Metabolites }\end{array}$ & $\begin{array}{c}\text { Maximum } \\
\text { RSD [\%] }\end{array}$ & $\begin{array}{c}\text { Average } \\
\text { RSD [\%] }\end{array}$ \\
\hline PM 1_Dilution with water & 52 & 30 & 9 \\
PM 2_Dilution with methanol & 52 & 62 & 8 \\
PM 3_Dilution with acetonitrile & 53 & 50 & 7 \\
PM 4_Freeze-drying, solution in 50\% methanol & 54 & 160 & 57 \\
PM 5_Filtration only & 56 & 25 & 8 \\
\hline
\end{tabular}

PM: preparation method; RSD: Relative standard deviation.

\subsection{Metabolite Profiles from Targeted Liquid Chromatography-High-Resolution Mass Spectrometry of} Skin Mucus

The ideal mucus sampling procedure would be the least invasive and at the same time allow for reproducible detection of as many metabolites as possible. Of the 86 target metabolites included in the present study, a total number of 60 were detected in the scraped, wiped, and absorbed mucus samples (Figure 1 and Table S2).
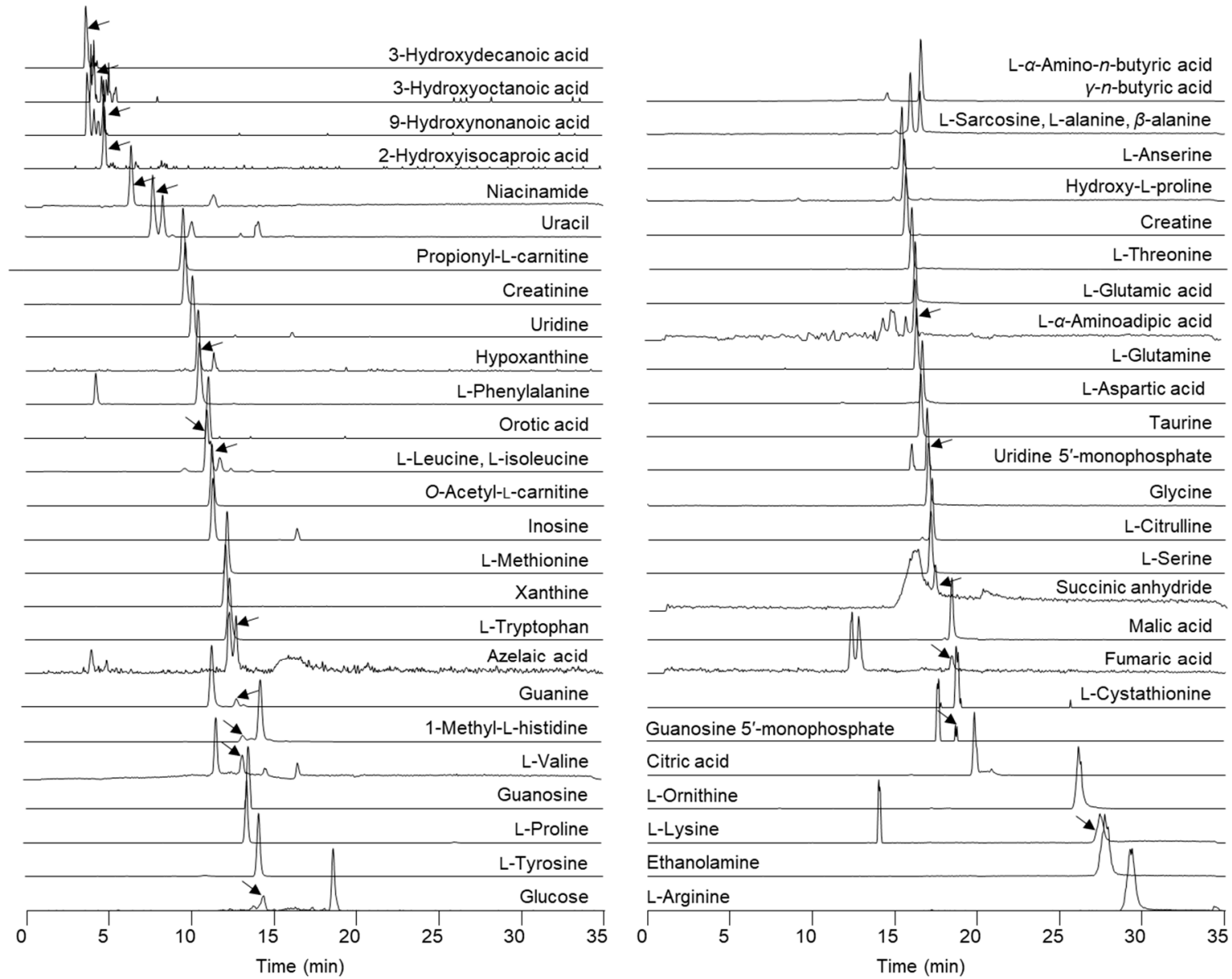

Figure 1. Extracted ion chromatograms from LC-HRMS ( $\pm 5 \mathrm{ppm})$ of salmon skin mucus collected using the absorption method. The figure is a collage of ion chromatograms from two different fish. 
Seven additional metabolites were detected in only few samples and with low signal/noise ratio. Even though the compounds' $\mathrm{m} / \mathrm{z}$ and retention times matched those of the reference standards, their further verification by HRMS/MS was not possible, and they were thus not included in the data set. These metabolites were butylated hydroxyanisole, 2-aminocaprylic acid, $N$-acetyl-L-alanine, L- $\beta$-aminoisobutyric acid, N $\alpha$-acetyl-L-arginine, $\alpha$-hydroxyadipic acid and L-cystine. An additional data-filtering step prior to multivariate analyses included the removal of metabolites that were detected in less than $80 \%$ of the mucus samples. Thus, 3-hydroxydecanoic acid, 3-hydroxyoctanoic acid, uracil, orotic acid, cytosine, 1-methyl-L-histidine, guanosine-5'-monophosphate, both oxidized and reduced L-glutathione, and $\delta$-hydroxylysine were detected in less than $80 \%$ of the samples. Furthermore, peak areas of guanine were of especially high variability between samples within the same sampling regime (relative standard deviation (RSD) up to $271 \%$ in absorbed mucus samples, $n=12$ ), and the metabolite was thus excluded from the data set prior to multivariate statistics. The variability of the absolute peak areas of the remaining 49 target metabolites between individuals of the same sampling regime was likewise relatively high, possibly as a result of differences in the hydration of the mucus during sampling. Thus, the highest variation in the overall absolute peak area for the 49 metabolites was observed in the filter absorbed mucus (79\% RSD, $n=12)$, while the overall peak areas for the 49 metabolites obtained from scraped and wiped mucus was somewhat lower (63\% and $62 \%$ RSD, respectively; $n=12)$. In contrast, the overall peak area for the 49 metabolites in the quality control $(\mathrm{QC})$ sample varied with 7\% RSD. In order to reduce this biased variation and enable comparison of the metabolite profiles obtained from the different sampling techniques, individual metabolite signals were normalized to the sum of all peak areas extracted for a given sample ("normalization by sum") [12]. Treating of the data in this way did not change the overall profile for the target metabolites (Figure 2).

Normalization by sum reduced the inter-individual variations for target metabolites within the same sampling regime. Principal component analysis (PCA) showed that individual scores from the scraped mucus samples and the absorbed samples partly clustered on opposite sides, while the scores from the wiped mucus clustered more or less between the two sets of observations (Figure 3).

However, the total variation of the target metabolites within each sampling group was similar, which can best be assessed from the Euclidian distance in the empirical cumulative distance plot (Figure 3). Several amino acids and xanthines as well as uridine were detected in considerably higher relative concentrations in scraped mucus as compared to wiped and absorbed mucus, whereas hydroxylated fatty acids (including 3-hydroxy-fatty acids that were detected in less than $80 \%$ of the fish) were detected in larger relative amounts in absorbed skin mucus compared to scraped mucus (Figure 2).

\subsection{Untargeted Liquid Chromatography-High-Resolution Mass Spectrometryof Fish Skin Mucus}

Processing of the LC-HRMS raw data set using MZmine version 2.10 (VTT Group, Espoo, Finland) including removal of background contaminants gave 1500 metabolic features from positive ionization and 1045 metabolic features from negative ionization. As discussed above, the dilution status of individual mucus samples, especially between samples obtained using the different sampling techniques, was expected to vary. We thus normalized the data by conversion of the absolute peak areas for each metabolic feature into relative peak areas by calculating the per cent contribution of each metabolic feature to the total peak area (obtained from summarization of the peak areas for all features in a sample). This data was applied to PCA in order to reduce the dimensions of the data set and visualize their maximum variation (Figure 4). 


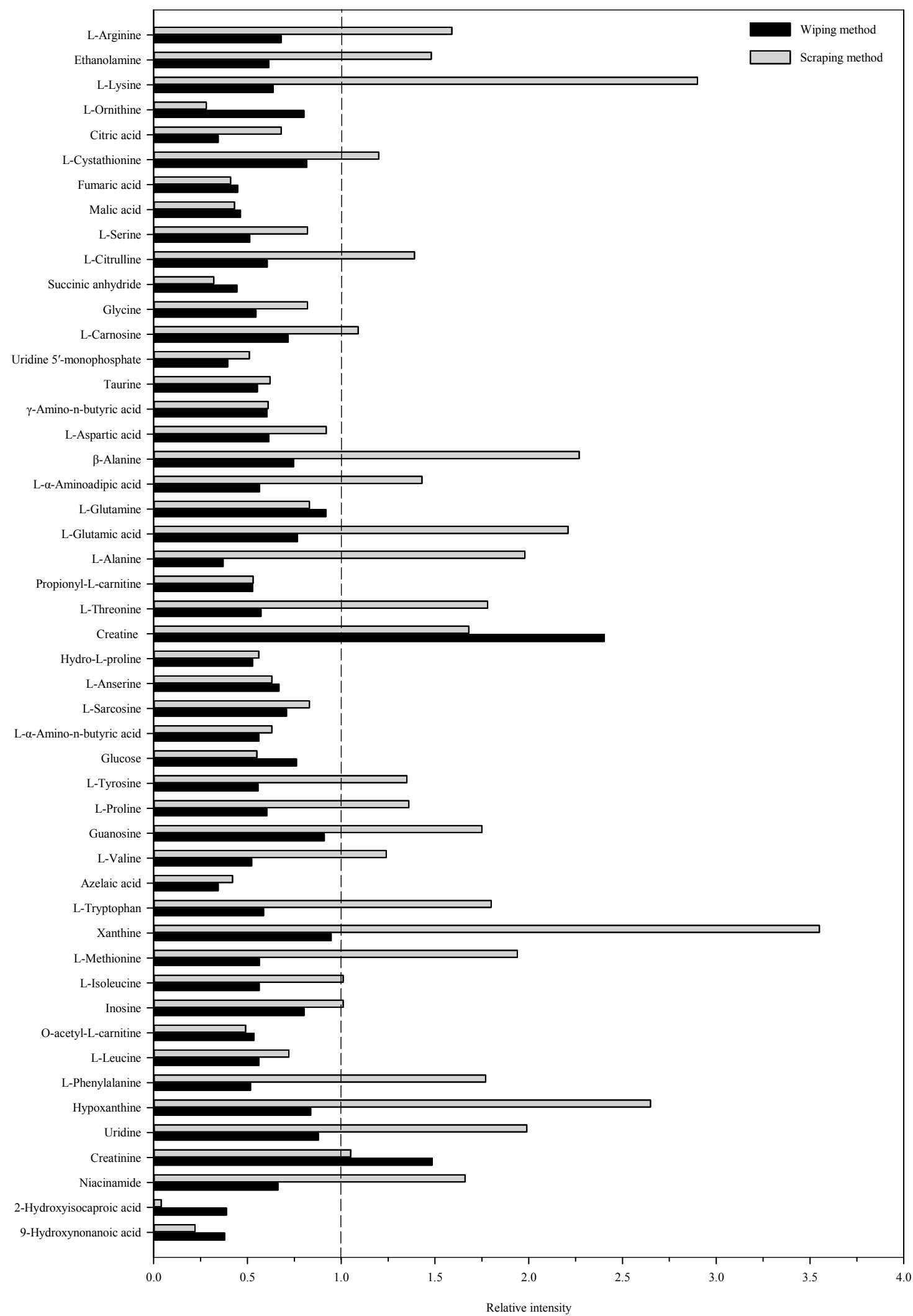

Figure 2. Comparison of mean peak areas from normalization by sum of target metabolites from salmon skin mucus, which was collected with three different sampling methods. The relative mean peak areas in the samples obtained with the filter absorption method were set to 1 (dashed line). The plot shows the variation of mean peak areas of individual metabolites between different sampling methods. 

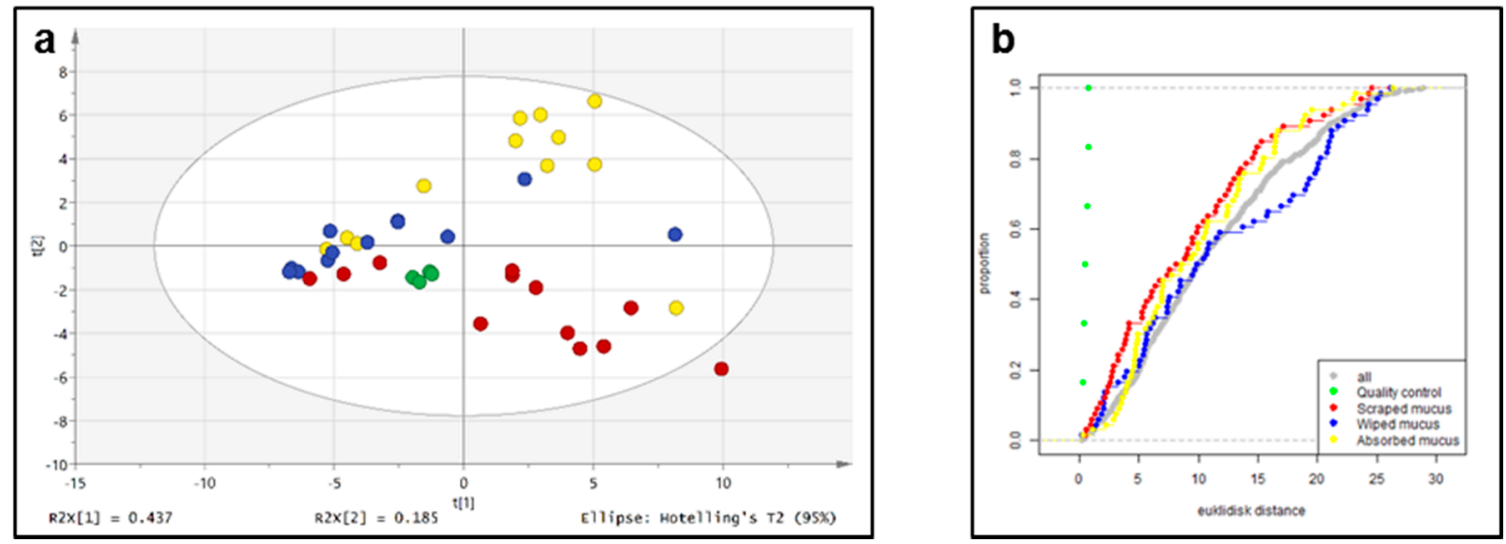

Figure 3. Score plot from principal component analysis (PCA) (a, unit variance scaled) of the metabolite profiles obtained from targeted LC-HRMS (including 49 metabolites) and corresponding cumulative Euclidic distance plot of the same data (b); quality control samples in green, scraped mucus samples in red, wiped mucus samples in blue, and absorbed mucus samples in yellow. The two first principal components explain $62 \%$ of the total variation.
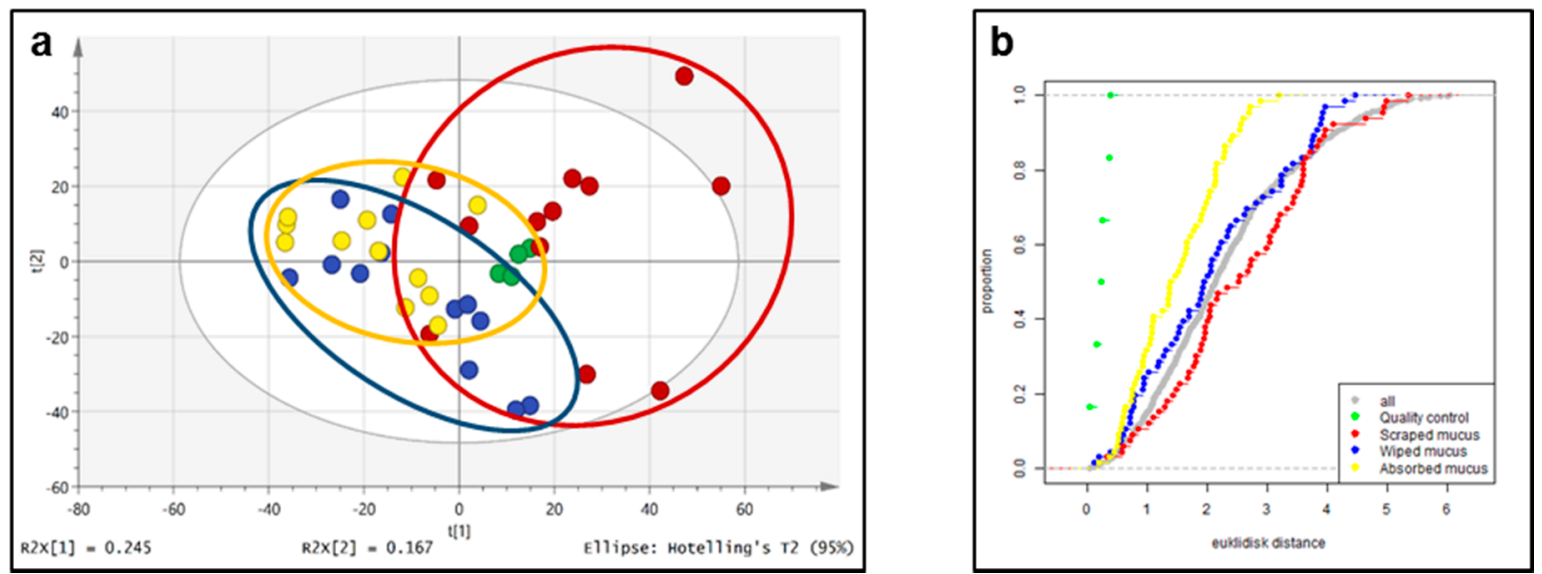

Figure 4. Score plot from PCA (a, unit variance scaled) of the metabolite profiles obtained from untargeted LC-HRMS and corresponding Euclidic distance plot of the same data (b); quality control samples in green, scraped mucus samples in red, wiped mucus samples in blue, and filter absorbed mucus samples in yellow. The two first components explain $41 \%$ of the total variation.

There exist several scaling methods for PCA. Here we used unit variance scaling (also called autoscaling), which has been shown to perform well for PCA of biological data [13]. The score plot shows that the total variation of the detected metabolite peak areas was lowest in the sample set obtained with the absorption method, while it was intermediate for the metabolite peak areas obtained using the wiping procedure and highest using the scraping method (Figure 4). The plot of the empirical cumulative distribution (Figure 4) showed that the Euclidian distance between samples where the absorbed mucus procedure was used was much more similar than the distance between those samples that were obtained with scraping. Importantly, the four QC features (pooled sample injected repeatedly throughout the analysis) cluster closely to each other in the middle of the PCA score plot, confirming excellent system stability. In addition, the global metabolite data obtained with the scraping method appeared to be rather different from the data obtained using the wiping and absorption method as the observations for the former tended to cluster into opposite parts of the PCA plane. Since comparative metabolomics studies require rather homogeneous samples with as little sampling-related bias as possible, the absorption method appears to be the method of choice based on the above data. 


\subsection{Targeted Feature Detection Using Thermo Xcalibur versus MZmine}

As reported above, 60 of the 86 target metabolites were detected using the semi-automatic re-processing function built in the Xcalibur software version 2.3 (Thermo Fisher Scientific, Waltham, MA, USA). After several filtering steps, the total number of detected peaks included in statistical models was reduced to 49 . Out of these 49 semi-automatically detected peaks, 47 were detected and extracted during fully automatized, untargeted processing using MZmine. Succinic anhydride and 2-hydroxyisocaproic acid were not covered by the MZmine dataset. The reason for the slightly lower number of target metabolites from MZmine could be attributed to the minimum peak intensity setting that was at $5 \times 105$ and/or the minimum ratio of peak top/edge (MRP) used during data processing, which was set to 5 [14]. Furthermore, for seven of the 47 metabolites, MZmine erroneously detected and extracted the 13C isotope peak (niacinamide, hypoxanthine, L- $\alpha$-amino- $n$-butyric acid, $\gamma$-amino- $n$-butyric acid, malic acid, ethanolamine, L-arginine). Unit variance scaled PCA was used in order to compare the target-metabolite profiles obtained with the two processing methods (Figure 5).

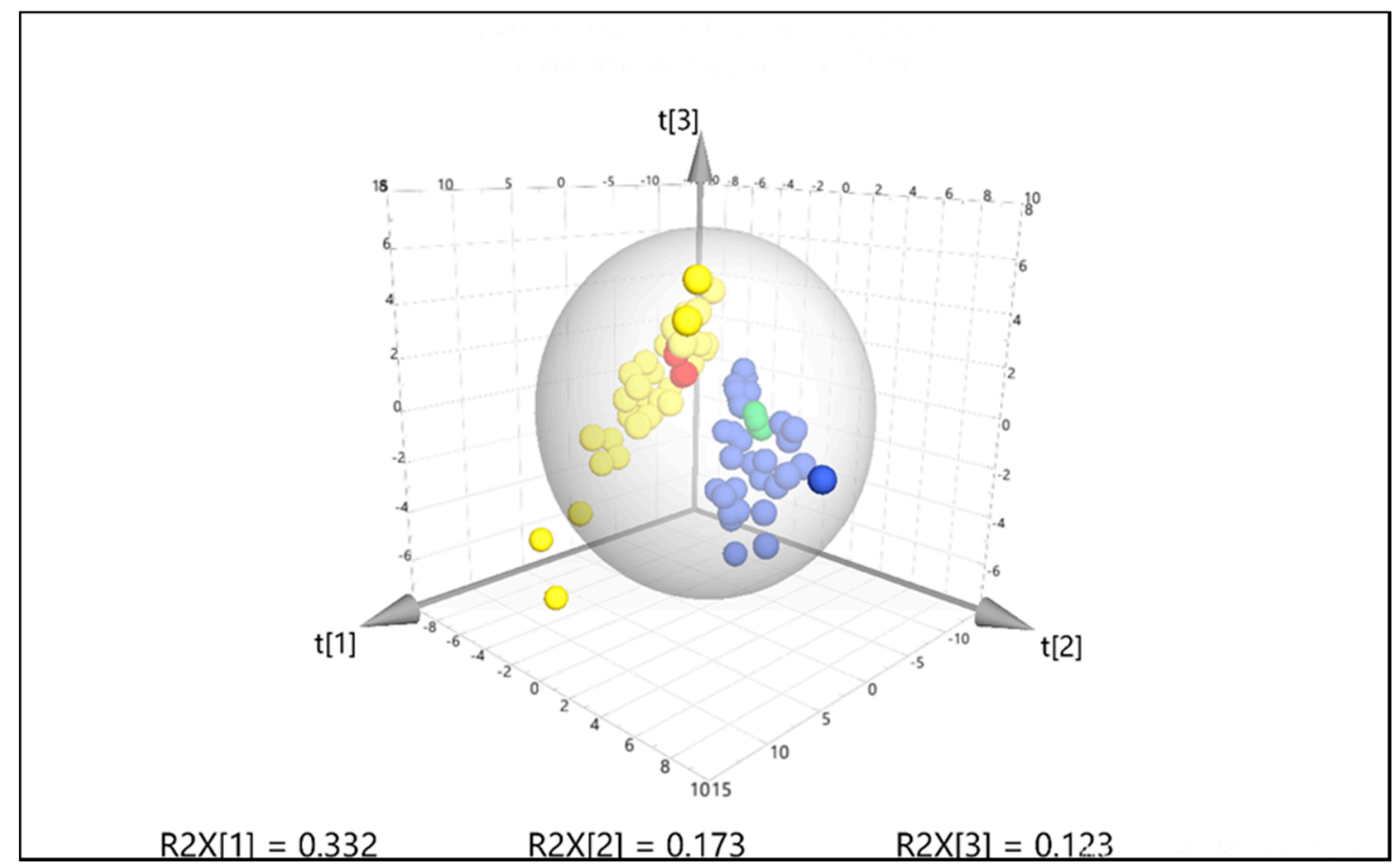

Figure 5. 3D-Score plot from PCA (unit variance scaled) of the profiles of 47 metabolites in all 36 mucus samples (without grouping according to sampling method) obtained from targeted LC-HRMS. The raw data were either processed using Thermo Fisher Xcalibur (samples in blue, Quality Control (QC) samples in green) or MZmine (samples in yellow, QC samples in red). The first three principal components explain $63 \%$ of the total variation.

The score scatter plot shows that the two data sets were different. The corresponding loading plots (Figure S3) revealed that the observed difference was (at least in part) a result of MZmine picking the $13 \mathrm{C}$ isotope peaks during data preprocessing. This is plausible since the $[\mathrm{M}+1]$ ion peak accounts merely for a few per cent of the peak intensity of the molecular ion resulting in a substantially different metabolite profile. Missing peaks as well as false positives from software tools using the centWave algorithm for detecting chromatographic peaks, such as MZmine or XCMS (Scripps Research Institute, La Jolla, CA, USA) have also been reported by other groups and will hopefully be debugged in future versions [12]. 


\subsection{Significance of Verified Mucosal Metabolites}

Fish mucus plays an important role in many biological functions for the fish such as respiration, reproduction, osmotic regulation, communication, and even as food in the parental care in some fish species [15]. Such functional diversity is reflected by the existence of a broad spectrum of molecules in mucus, including metabolites. Though metabolites in fish skin mucus has received relative little attention, previous studies have identified different classes of metabolites, including free fatty acids, free amino acids, organic acids, purines, and pyrimidines [10].

The fish epidermal mucus constitutes a physical interface between fish and environment. The molecular composition of mucus, including its metabolite profile, will therefore reflect not only specific components from the fish but also molecules from (organisms in) the surrounding aquatic environment and the microbiota colonizing the mucosal surface [16]. Mucus is also the first defensive barrier that pathogens have to breach or inhabit during infection. The mucus is accordingly rich in defensive (immune) molecules of fish origin and a likely variable population of pathogen- and microbiota-derived components [17].

In the present study, both targeted and untargeted analysis showed that the scraping method resulted in metabolite profiles that were relatively distinct from those of the filter absorption and wiping methods. This is likely due to the comparative invasive nature of the scraping method, where scales and epidermal cells inevitably will be compromised. Metabolites leaking from the affected tissue will contribute to the sample, which consequently could lead to a relative higher ratio of host-derived metabolites. Another explanation of the observed difference in the metabolite profiles between sampling methods could be that some of the metabolites remained partially adsorbed to the wipes. The fact that the filter absorption method produced samples with relatively higher contents of 3-hydroxylated fatty acids (Figure 2), which are likely microbe-derived components [18], could suggest that this sample type contains comparatively more non-host factors. Absorbed samples contain a minimum of mucus matrix and associated host components and this may explain this relative difference. The detected fatty acids 9-hydroxy-nonanoic acid and 2-hydroxy-isocaproic acid (Figure 2 and Table S2) could be host-derived [10] and as such play a role in the defensive system. Free fatty acids have been shown to have potent anti-bacterial effects, likely by interfering with the bacterial membrane, though disruption of electron transport chain, uncoupling of oxidative phosphorylation, inhibition of enzyme activity, peroxidation and auto-oxidation, and cell lysis could all contribute [19]. In a study from 1970, Lewis [20] found the free fatty acids to be a prominent part of mucosal lipids in fish and suggested that they could contribute to protection against bacterial and fungal diseases as they do in human sebum [21]. Along with cholesterol and ceramides, free fatty acids play a crucial role in skin barrier functions in mammals [21]. The mucosal free fatty acids in fish could result from lipolysis of triglycerides [20]. Despite a scarcity of studies, a few relatively recent reports have confirmed the detection of free fatty acids in fish skin mucus of different species $[10,20,22]$.

Another important feature of fish epidermal mucus is the occurrence of free amino acids, which have previously been demonstrated in a number of fish species [10,23-26]. In the targeted analysis in the present study, several free amino acids were detected in fish mucus (Figure 2 and Table S2), which largely confirms the findings of Ekman et al. [10] in epidermal mucus from fathead minnow (Pimephales promelas). In the European eel (Anguilla anguilla), the similarity of amino acid patterns between mucosal free amino acids and composition of mucosal proteins suggested that degradation of mucosal proteins could be a potential origin of mucosal free amino acids [27]. Several studies suggest that levels of mucosal free amino acids are not stable but tend to fluctuate in response to age, fish nutrition and season $[25,27,28]$. The functional role of mucosal free amino acids and their biotransformation products are likely diverse and includes osmoreagulation (taurine) [27,29], olfaction $[23,25]$ as well as wound healing [30,31]. Arginine and L-ornithine, identified in the targeted analysis in the present study, have been suggested to contribute to skin health and wound healing processes in mammals $[30,31]$. An improved understanding of how mucosal amino acids contribute in 
wound healing in fish would generally be beneficial to aquaculture which is severely affected by ulcer and wound-causing infectious diseases.

Fish mucus also contains nucleosides, nucleotides, and their precursors and derivatives [10], which was confirmed in the current study (Table S2). In mammals, extracellular nucleotides like adenosine triphosphate (ATP) and uridine-5'-triphosphate (UTP) were found to regulate the mucus clearance over different mucosal surfaces, which consequently protects against pathogenic damages [32,33]. Except for a study of the importance of purines and pyrimidines in the process of ammonia excretion [34], this group's metabolites have not yet been studied in fish mucus. Future investigation of the potential role of metabolites in the clearance of mucus-pathogen complexes of the fish mucosal surfaces should be pursued.

\section{Materials and Methods}

\subsection{Materials}

LC-MS grade water and acetonitrile were from Fisher Scientific (Oslo, Norway), high performance liquid chromatography (HPLC)-grade methanol from Romil (Cambridge, UK), dimethyl sulfoxide (DMSO, pro analysis quality) was from Merck KGaA (Darmstadt, Germany), whereas ammonium carbonate was from Fluka (Steinheim, Germany). All reference compounds for the 89 metabolites were from Sigma-Aldrich (St. Louis, MO, USA), and stock solutions prepared in water, $1 \mathrm{M}$ sodium hydroxide, water/methanol, methanol, or DMSO (Table S1).

\subsection{Fish Breeding}

The study was conducted in accordance with the Declaration of Helsinki, and the protocol was approved by the Norwegian Animal Research Authority (FOTS ID 12009).

Atlantic salmon (Salmo salar) $(n=24)$ with an average body weight of $1 \mathrm{~kg}$ were obtained from the Drøbak Research Station of the Norwegian Water Research Institute, Norway. Three months before the experiment, fish were vaccinated with ALPHA JECT micro $6{ }^{\circledR}$ (PHARMAQ AS, Overhalla, Norway) vaccine $(100 \mu \mathrm{L} /$ fish $)$ and were pit-tagged to facilitate correlation of individual fish to their sequential mucus samples.

\subsection{General Mucus Sampling Procedure}

All mucus samples were obtained between 10 a.m. and 2 p.m. Only fish with apparently healthy skin and clear mucus were included in this study, and fish showing signs of external lacerations were excluded. Prior to sampling, fish were pre-anesthetized with $10 \mathrm{mg} / \mathrm{L}$ of benzocaine (ACD Pharmaceuticals AS, Oslo, Norway) in the holding tanks to minimize handling-induced disturbance of the mucus layer, anesthetized with benzocaine $(200 \mathrm{mg} / \mathrm{L})$ for five minutes in a separate container, scanned for their pit-tags, and then left for $10 \mathrm{~s}$ to trickle the extra water. In order to collect as much mucus as possible, anesthetized fish were positioned on their ventral sides (no mucus samples were collected from the ventral side), and fish were sampled from both right and left sides.

\subsection{Epidermal Mucus Sampling}

Three different mucus sampling methods (defined below and hereafter named absorption, wiping, and scraping methods, respectively) were used pairwise on individual fish to collect epidermal mucus sample pairs from 24 fish (Figure 6). The fish were randomly divided into three groups (Group 1-3) of eight fish. From each fish in Group 1, mucus was sampled by scraping from one entire lateral side and by wiping from the opposite lateral side. In Group 2, the sampling methods were scraping and absorption, respectively, and in Group 3, absorption and wiping. 


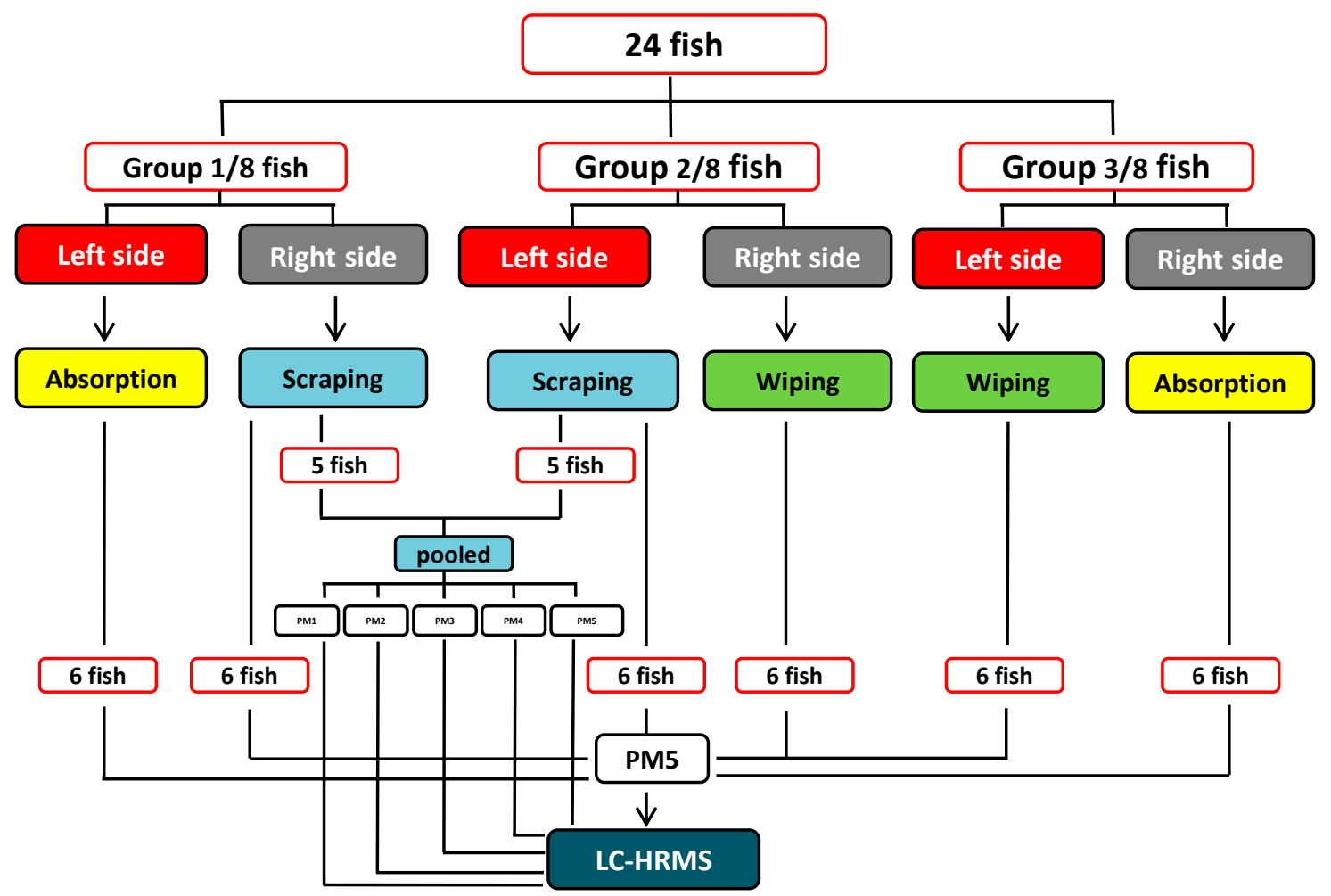

Figure 6. Schematic flow diagram of epidermal mucus sampling.

\subsubsection{Absorption Method}

The 16 fish assigned to this method in Groups 2 and 3 (Figure 6) were placed on the ventral side. One of the lateral sides (randomly left or right) of each fish was covered with sterile pieces $(2.5 \times 7 \mathrm{~cm} /$ each) of white medical wipes (Klimberly-Clark, Irving, TX, USA) to absorb the water phase of the mucus. After absorption, the wipes were gently removed with forceps and were placed into the upper compartments of several Spin- $X^{\circledR}$ polypropylene centrifuge tubes $(0.22 \mu \mathrm{m}$, Costar, Corning, NY, USA) and stored on ice until the tubes were centrifuged.

\subsubsection{Wiping Method}

The mucus layer from one of either side of all 16 fish in Groups 1 and 3 (Figure 6) was directional wiped off (i.e., from head toward tail) using the same medical wipes as were used in the absorption method. The tissue paper pieces were thereafter placed into the upper compartments of several Spin- $X$ centrifuge tubes as above and stored on ice until the tubes were centrifuged.

\subsubsection{Scraping Method}

The mucus layer from one of either side of all 16 fish in Groups 1 and 2 (Figure 6) was directional scraped off (i.e., in the direction of the scales) with a sterile and blunt back of a scalpel blade. The scraped samples were funneled into a plastic tray and subsequently transferred into the upper compartments of Spin- $X$ centrifuge tubes as above stored on ice until the tubes were centrifuged.

\subsection{Collection of Mucus Fluid}

Mucus fluid was obtained from the medical wipes (absorption and wiping methods) by centrifugation of the Spin-X tubes at $500 \times g$ for $10 \mathrm{~min}\left(4^{\circ} \mathrm{C}\right)$. The scraped mucus samples were treated in the same way in order to shred the viscous mucus. The mucus fluid samples were then collected from the lower compartments of the Spin- $X$ tubes and stored at $-80{ }^{\circ} \mathrm{C}$ until further analysis. 


\subsection{Sample Preparation}

In order to evaluate different sample pretreatment protocols a pooled scraped mucus sample was obtained from ten randomly selected fish. Five different procedures were tested for sample preparation prior to LC-MS analysis. The effect of mucus dilution (PM 1-3) and freeze drying (PM 4) were evaluated and compared to the direct analysis method described by Ræder et al. (2007) (PM 5) [35]. PM 1-3 consisted of addition of either water, methanol or acetonitrile (all 1:1, v/v), respectively, to an aliquot of pooled scraped mucus $(150 \mu \mathrm{L})$ in triplicate. In PM 4, $150 \mu \mathrm{L}$-aliquots were freeze-dried and then reconstituted in $150 \mu \mathrm{L}$ of $50 \%$ methanol in water. After vortex mixing for $30 \mathrm{~s}$, all of the above resulting mixtures (PM 1-4) were centrifuged at $14,000 \times g$ for $15 \min$ at $4{ }^{\circ} \mathrm{C}$ and then filtered (Costar Spin-X, $0.22 \mu \mathrm{m})$. In PM 5, mucus aliquots were centrifuged $(14,000 \times g$, $15 \mathrm{~min}$ at $4{ }^{\circ} \mathrm{C}$ ) and filtered (Spin-X) prior to analysis [35]. Triplicate samples were prepared for each pretreatment method.

\subsection{Liquid Chromatography-High-Resolution Mass SpectrometryData Acquisition}

Samples were placed randomly in the autosampler tray, which was thermostatted to $20{ }^{\circ} \mathrm{C}$. The instrument used was a Thermo Fisher Scientific (Bremen, Germany) Vanquish UHPLC interfaced to a Q-Exactive Fourier-transform high-resolution mass spectrometer, equipped with a heated electrospray interface. A zwitterionic SeQuant ZIC-pHILIC column (Merck, Kenilworth, NJ, USA; $150 \times 4.6 \mathrm{~mm}, 5 \mu \mathrm{m}$ ) was employed for HILIC. The mobile phases used for HILIC were $20 \mathrm{mM}$ ammonium carbonate (A, pH 8.3) and pure acetonitrile (B). The column was eluted isocratically for one min using $80 \% \mathrm{~B}$, followed by linear gradient elution to $20 \% \mathrm{~B}$ in $29 \mathrm{~min}$. After flushing the column with $8 \% \mathrm{~B}$, the mobile phase composition was returned to starting conditions and equilibrated for $9 \mathrm{~min}$. The mass spectrometer was run in positive and negative ion full-scan mode using fast polarity switching (i.e., alternating positive and negative ion scans), in the mass range $m / z 58-870$. The mass resolution was set to 70,000 at $m / z 200$. The spray voltage was 2.8 and $3.2 \mathrm{kV}$ (positive and negative mode, respectively), the transfer capillary temperature was $280^{\circ} \mathrm{C}$, and the sheath and auxiliary gas flow rates were 35 and 10 units, respectively. Xcalibur software was used for instrument control and calculation of mass errors and elemental compositions.

The identity of target metabolites eluting at slightly different retention times in the mucus samples relative to the reference standard solutions or those that showed nearby eluting compounds with similar $m / z$ was verified using parallel reaction monitoring (targeted HRMS/MS). Precursor ions were selected in the quadrupole with an isolation width of $3 \mathrm{~m} / z$ and fragmented using higher-energy collision dissociation and normalized collision energy of $40 \%$. The mass resolution for scanning of the product ions was set to 17,500 .

\subsection{Raw Data Processing Using MZmine}

The raw data was split into a single positive and negative ion data set, and also converted into mzML format using ProteoWizard [36]. MZmine version 2.10 was used for processing of the dataset, and the settings for each step are described in Table S3 [37]. A pooled QC sample was prepared along with the test samples and was measured periodically throughout the whole LC-HRMS experiment. Four sets of QC data were acquired to assess system stability. In addition, a blank sample was injected periodically throughout the LC-HRMS run, and features detected in the blank analyses removed from the sample peak list. Based on this filtered peak list, the data for individual test samples were aligned, and all features not matching with the pooled sample peak list were removed.

\subsection{Multivariate Analysis}

Multivariate statistical analyses were performed in SIMCA (Umetrics, Sartorius Stedim Biotech, Malmö, Sweden) and R [38]. For normalization of metabolite patterns, the percent peak area for each metabolic feature was calculated from the total peak area for each sample. Prior to PCA, the data 
were mean-centered and unit variance scaled. Individual peak areas were normalized using the normalization-by-sum approach (normalization of the data by conversion of the absolute peak areas for each metabolic feature into relative peak areas by calculating the per cent contribution of each metabolic feature to the total peak area). PCA score plots were assessed based on the first two and three principal components. The Euclidian distances between all pairs of fish based on the two first principal components were calculated, and an empirical cumulative distribution was plotted for fish pair samples originating from the same groups.

\section{Conclusions}

The workflow presented here represents the groundwork for future metabolomics studies in fish health and welfare studies e.g., studies into host-parasite interactions or the effect of certain feeding regimes on mucus composition. We show that a little invasive sampling method combined with minimal sample preparation and subsequent LC-HRMS analysis is well suited for the targeted and untargeted analysis of polar to medium polar metabolites in fish skin mucus. Using our approach, we were able to verify 60 metabolites in the skin mucus of salmon. The majority of these were amino acids and small organic acids and are likely fish metabolites, while other, such as 3-hydroxy fatty acids, could be related to microbes (e.g., Gram-negative bacteria).

Supplementary Materials: The following are available online at http:/ www.mdpi.com/2410-3888/3/2/21/s1, Figure S1: 3D-Score plot from principal component analysis (PCA) of the metabolite profiles obtained from untargeted LC-HRMS comparing the total variation in the mucus samples collected by scraping, wiping or absorption; Figure S2: Loading plots from PCA of the profiles of 47 metabolites in all 36 mucus samples, processed either using MZmine 2 or Xcalibur; Table S1: Complete overview over reference compounds; Table S2: LC-HRMS characteristics of all 89 small metabolites; Table S3: MZmine 2 processing parameters .

Author Contributions: S.U. and S.G. designed and supervised the study. H.T. and S.G. performed the sampling. L.I. and S.U. performed analysis, processed the raw data and wrote the manuscript with input from H.T., S.G. and A.B.K.; A.B.K. provided expertise in statistical analyses and interpretation of collected data. All authors have read, revised, and approved the final version of the manuscript.

Funding: This research was funded by The Research Council of Norway (grant number 235477) and the Norwegian Ministry of Trade, Industry and Fisheries.

Conflicts of Interest: The authors declare no conflict of interest. The founding sponsors had no role in the design of the study; in the collection, analyses, or interpretation of data; in the writing of the manuscript, and in the decision to publish the results.

\section{References}

1. Food and Agriculture Organization of the United Nations. The State of World Fisheries and Aquaculture 2016: Contributing to Food Security and Nutrition for All. Available online: http: / wwwfaoorg/3/a-i5555epdf (accessed on 7 July 2016).

2. Esteban, M.A. An overview of the immunological defenses in fish skin. ISRN Immunol. 2012, 2012, 853470.

3. Easy, R.H.; Ross, N.W. Changes in Atlantic salmon (Salmo salar) epidermal mucus protein composition profiles following infection with sea lice (Lepeophtheirus salmonis). Comp. Biochem. Physiol. Part D Genom. Proteom. 2009, 4, 159-167. [CrossRef] [PubMed]

4. Guardiola, F.A.; Cuesta, A.; Abellán, E.; Meseguer, J.; Esteban, M.A. Comparative analysis of the humoral immunity of skin mucus from several marine teleost fish. Fish Shellfish Immunol. 2014, 40, 24-31. [CrossRef] [PubMed]

5. Subramanian, S.; MacKinnon, S.L.; Ross, N.W. A comparative study on innate immune parameters in the epidermal mucus of various fish species. Comp. Biochem. Physiol. Part. B. Biochem. Mol. Biol. 2007, 148, 256-263. [CrossRef] [PubMed]

6. Brinchmann, M.F. Immune relevant molecules identified in the skin mucus of fish using -omics technologies. Mol. Biosyst. 2016, 12, 2056-2063. [CrossRef] [PubMed]

7. Bruce, T.J.; Brown, M.L. A review of immune system components, cytokines, and immunostimulants in cultured finfish species. Open J. Anim. Sci. 2017, 7, 267-288. [CrossRef] 
8. Guardiola, F.A.; Cuartero, M.; Del Mar Collado-Gonzalez, M.; Diaz Banos, F.G.; Cuesta, A.; Morinigo, M.A.; Esteban, M.A. Terminal carbohydrates abundance, immune related enzymes, bactericidal activity and physico-chemical parameters of the Senegalese sole (Solea senegalensis, Kaup) skin mucus. Fish Shellfish Immunol. 2017, 60, 483-491. [CrossRef] [PubMed]

9. Haniffa, M.A.; Viswanathan, S.; Jancy, D.; Poomari, K.; Manikandan, S. Antibacterial studies of fish mucus from two marketed air-breathing fishes-Channa striatus and Heteropneustes fossilis. Int. Res. J. Microbiol. 2014, 5, 22-27.

10. Ekman, D.R.; Skelton, D.M.; Davis, J.M.; Villeneuve, D.L.; Cavallin, J.E.; Schroeder, A.; Jensen, K.M.; Ankley, G.T.; Collette, T.W. Metabolite profiling of fish skin mucus: A novel approach for minimally-invasive environmental exposure monitoring and surveillance. Environ. Sci. Technol. 2015, 49, 3091-3100. [CrossRef] [PubMed]

11. Zhang, R.; Watson, D.G.; Wang, L.J.; Westrop, G.D.; Coombs, G.H.; Zhang, T. Evaluation of mobile phase characteristics on three zwitterionic columns in hydrophilic interaction liquid chromatography mode for liquid chromatography-high resolution mass spectrometry based untargeted metabolite profiling of Leishmania parasites. J. Chromatogr. A 2014, 1362, 168-179. [CrossRef] [PubMed]

12. Di Guida, R.; Engel, J.; Allwood, J.W.; Weber, R.J.; Jones, M.R.; Sommer, U.; Viant, M.R.; Dunn, W.B. Non-targeted UHPLC-MS metabolomic data processing methods: A comparative investigation of normalisation, missing value imputation, transformation and scaling. Metabolomics 2016, 12, 93. [CrossRef] [PubMed]

13. Van den Berg, R.A.; Hoefsloot, H.C.J.; Westerhuis, J.A.; Smilde, A.K.; Van der Werf, M.J. Centering, scaling, and transformations: Improving the biological information content of metabolomics data. BMC Genom. 2006, 7, 142. [CrossRef] [PubMed]

14. Hu, M.; Krauss, M.; Brack, W.; Schulze, T. Optimization of LC-Orbitrap-HRMS acquisition and MZmine 2 data processing for nontarget screening of environmental samples using design of experiments. Anal. Bioanal. Chem. 2016, 408, 7905-7915. [CrossRef] [PubMed]

15. Shephard, K.L. Functions for fish mucus. Rev. Fish Biol. Fish. 1994, 4, 401-429. [CrossRef]

16. Reverter, M.; Sasal, P.; Banaigs, B.; Lecchini, D.; Lecellier, G.; Tapissier-Bontemps, N. Fish mucus metabolome reveals fish life-history traits. Coral Reefs 2017, 36, 463-475. [CrossRef]

17. Benhamed, S.; Guardiola, F.A.; Mars, M.; Esteban, M.Á. Pathogen bacteria adhesion to skin mucus of fishes. Vet. Microbiol. 2014, 171, 1-12. [CrossRef] [PubMed]

18. Maitra, S.K.; Nachum, R.; Pearson, F.C. Establishment of beta-hydroxy fatty acids as chemical marker molecules for bacterial endotoxin by gas chromatography-mass spectrometry. Appl. Environ. Microbiol. 1986, 52, 510-514. [PubMed]

19. Desbois, A.P.; Smith, V.J. Antibacterial free fatty acids: Activities, mechanisms of action and biotechnological potential. Appl. Microbiol. Biotechnol. 2010, 85, 1629-1642. [CrossRef] [PubMed]

20. Lewis, R.W. Fish cutaneous mucus: A new source of skin surface lipid. Lipids 1970, 5, 947-949. [CrossRef]

21. Smeden, J.; Janssens, M.; Kaye, E.C.J.; Caspers, P.J.; Lavrijsen, A.P.; Vreeken, R.J.; Bouwstra, J.A. The importance of free fatty acid chain length for the skin barrier function in atopic eczema patients. Exp. Dermatol. 2014, 23, 45-52. [CrossRef] [PubMed]

22. Jais, A.M.M.; Matori, M.F.; Kittakoop, P.; Sowanborirux, K. Fatty acid compositions in mucus and roe of Haruan, Channa striatus, for wound healing. Gen. Pharmacol. 1998, 30, 561-563. [CrossRef]

23. Hara, T.J.; Macdonald, S.; Evans, R.E.; Marui, T.; Arai, S. Morpholine, bile acids and skin mucus as possible chemical cues in salmonid homing: Electrophysiological re-evaluation. In Mechanisms of Migration in Fishes; Springer: Berlin, Germany, 1984; pp. 363-378.

24. Kallert, D.M.; Ponader, S.; Adelt, S.; Kaese, P.; Geyer, R.; Haas, W.; El-Matbouli, M. Analysis of rainbow trout Oncorhynchus mykiss epidermal mucus and evaluation of semiochemical activity for polar filament discharge in Myxobolus cerebralis actinospores. J. Fish Biol. 2010, 77, 1579-1598. [CrossRef] [PubMed]

25. Saglio, P.; Fauconneau, B. Free amino acid content in the skin mucus of goldfish, Carassius auratus L.: Influence of feeding. Comp. Biochem. Physiol. Part A Physiol. 1985, 82, 67-70. [CrossRef]

26. Stabell, O.B.; Selset, R. Comparison of mucus collecting methods in fish olfaction. Acta Physiol. 1980, 108, 91-96. [CrossRef] [PubMed]

27. Fauconneau, B.; Saglio, P. Protein-bound and free amino acid content in the skin mucus of the European eel, Anguilla anguilla (L.). Comp. Biochem. Physiol. B 1984, 77, 513-516. [CrossRef] 
28. Chong, K.; Ying, T.; Foo, J.; Jin, L.T.; Chong, A. Characterisation of proteins in epidermal mucus of discus fish (Symphysodon spp.) during parental phase. Aquaculture 2005, 249, 469-476. [CrossRef]

29. Shiau, C.-Y.; Pong, Y.-J.; Chiou, T.-K.; Chai, T.-J. Effect of growth on the levels of free histidine and amino acids in white muscle of milkfish (Chanos chanos). J. Agric. Food Chem. 1997, 45, 2103-2106. [CrossRef]

30. Shi, H.P.; Fishel, R.S.; Efron, D.T.; Williams, J.Z.; Fishel, M.H.; Barbul, A. Effect of supplemental ornithine on wound healing. J. Surg. Res. 2002, 106, 299-302. [CrossRef] [PubMed]

31. Wesley, J.A.; Supp, D.M. Role of arginine and omega-3 fatty acids in wound healing and infection. Adv. Wound. Care 2014, 3, 682-690.

32. Button, B.; Boucher, R.C. Role of mechanical stress in regulating airway surface hydration and mucus clearance rates. Respir. Physiol. Neurobiol. 2008, 163, 189-201. [CrossRef] [PubMed]

33. Ueda, F.; Watanabe, M.; Hirata, Y.; Kyoi, T.; Kimura, K. Changes in cyclic AMP content of rat gastric mucosa induced by ulcerogenic stimuli-In relation to the antiulcer activity of irsogladine maleate. Jpn. J. Pharmacol. 1991, 55, 493-499. [CrossRef] [PubMed]

34. Van Waarde, A. Biochemistry of non-protein nitrogenous compounds in fish including the use of amino acids for anaerobic energy production. Comp. Biochem. Physiol. B 1988, 91, 207-228. [CrossRef]

35. Ræder, I.L.U.; Paulsen, S.M.; Smalås, A.O.; Willassen, N.P. Effect of fish skin mucus on the soluble proteome of Vibrio salmonicida analysed by 2-D gel electrophoresis and tandem mass spectrometry. Microb. Pathog. 2007, 42, 36-45. [CrossRef] [PubMed]

36. Holman, J.D.; Tabb, D.L.; Mallick, P. Employing ProteoWizard to convert raw mass spectrometry data. Curr. Protoc. Bioinform. 2014, 46, 1-9.

37. Pluskal, T.; Castillo, S.; Villar-Briones, A.; Oresic, M. MZmine 2: Modular framework for processing, visualizing, and analyzing mass spectrometry-based molecular profile data. BMC Bioinform. 2010, 11, 395. [CrossRef] [PubMed]

38. The R Project for Statistical Computing Website. Available online: https://www.r-project.org/ (accessed on 6 May 2018).

(C) 2018 by the authors. Licensee MDPI, Basel, Switzerland. This article is an open access article distributed under the terms and conditions of the Creative Commons Attribution (CC BY) license (http:/ / creativecommons.org/licenses/by/4.0/). 